\title{
Determination of time-dependent uncertainty of the total solar irradiance records from 1978 to present
}

\author{
Claus Fröhlich* \\ Dählenwaldstrasse 30, 7265 Davos Wolfgang, Switzerland \\ ${ }^{*}$ Corresponding author: cfrohlich@pmodwrc.ch
}

Received 30 December 2014 / Accepted 5 February 2016

\begin{abstract}
Aims. The existing records of total solar irradiance (TSI) since 1978 differ not only in absolute values, but also show different trends. For the study of TSI variability these records need to be combined and three composites have been devised; however, the results depend on the choice of the records and the way they are combined. A new composite should be based on all existing records with an individual qualification. It is proposed to use a time-dependent uncertainty for weighting of the individual records. Methods. The determination of the time-dependent deviation of the TSI records is performed by comparison with the square root of the sunspot number (SSN). However, this correlation is only valid for timescales of the order of a year or more because TSI and SSN react quite differently to solar activity changes on shorter timescales. Hence the results concern only periods longer than the one-year-low-pass filter used in the analysis.

Results. Besides the main objective to determine an investigator-independent uncertainty, the comparison of TSI with $\sqrt{\mathrm{SSN}}$ turns out to be a powerful tool for the study of the TSI long-term changes. The correlation of $\sqrt{\mathrm{SSN}}$ with TSI replicates very well the TSI minima, especially the very low value of the recent minimum. The results of the uncertainty determination confirm not only the need for adequate corrections for degradation, but also show that a rather detailed analysis is needed. The daily average of all TSI values available on that day, weighted with the correspondingly determined uncertainty, is used to construct a "new" composite, which, overall, compares well with the Physikalisch-Meteorologisches Observatorium Davos (PMOD) composite. Finally, the TSI $-\sqrt{\text { SSN }}$ comparison proves to be an important diagnostic tool not only for estimating uncertainties of observations, but also for a better understanding of the long-term variability of TSI.
\end{abstract}

Key words. Total solar irradiance - Solar activity - Solar variability - Total solar irradiance composite

\section{Introduction}

From Figure 1 it is obvious that for any long-term analysis of total solar irradiance (TSI) for solar physics or Earth's climate research, a combination of all these records is needed. Three TSI composites were constructed by the teams of the Active Cavity Radiometer for Irradiance Monitoring (ACRIM), the Institut Royal Météorologique de Belgique (IRMB) and the Physikalisch-Meteorologisches Observatorium Davos (PMOD; see e.g. Dewitte et al. 2004; Fröhlich 2006; Willson 2014). They are based on a selection of the TSI records since 1978 (Fig. 1), which were then adjusted during their overlapping periods and referenced to the absolute value of one of the radiometers. The long-term changes due to the exposure in space called degradation - of HF/Nimbus-7 could not be removed in the original analysis and the degradation of ACRIM1 was overestimated. Hence, Fröhlich (2006) developed corrections for both time series, which were then used for constructing the PMOD composite, whereas the other composites use the original data. So, there are important differences during the maximum and the descending part of solar cycle 21, and during the period between the end of ACRIM1 and the beginning of ACRIM2 - the so-called ACRIM gap - which depend on which time series is used to bridge this gap (for a detailed discussion see e.g. Fröhlich 2012).

For the construction of a new, objective composite an independent method is needed to judge the uncertainty of the different TSI records - the original and corrected ones - which then can be used to weight the individual TSI records accordingly. In Section 2, a method is described to determine a timedependent uncertainty based on the standard deviation relative to a reference, while Section 3 presents the construction of a "new" composite TSI.

\section{Determination of a time-dependent uncertainty of the TSI records}

To generate a new composite TSI time series out of multiple records, we need a time-dependent weighting of these records. Ideally this weighting should depend on the difference between each TSI record and an independent reference time series, which needs to have a similar reaction to the temporal changes of solar activity as TSI. The sunspot number (SSN, version $2.0^{1}$ ) seems a valuable candidate. However, the correlation between daily values of TSI and SSN is rather low; this is due to the fact that during the passage of active regions the daily SSN depends only on the number of spots present on the disc that day, while daily TSI values depend on a combination of sunspot darkening and facular brightening. On the other hand, SSN and TSI show a similar dependence on the strength of solar activity on timescales of one year or more. Ångström (1922) was the first to correlate the solar constant

\footnotetext{
${ }^{1}$ From WDC-SILSO, Royal Observatory of Belgium, Brussels (for details see e.g. Clette et al. 2014).
} 
of Abbot (1913) with the square root of SSN rather than with SSN directly. Indeed, for a time series that follows a Poisson distribution, taking the square root results in a time series, which is approximately normally distributed (e.g. Anscombe 1948), and more importantly, approaches zero with a non-zero slope, which allows to determine a unique TSI value at $\mathrm{SSN}=0$, hereafter designated as offset $S_{0}$. Moreover, Wang $\&$ Sheeley (2003) showed that the fluctuations of the equatorial magnetic field, as e.g. represented by $B$ at $1 \mathrm{AU}$, scale with the square root of the emergence rate of active regions, which is proportional to SSN. Svalgaard \& Cliver (2007) showed that this relationship holds over the past 12 solar cycles, and concluded that $B$ must have a "floor" as it cannot go below $\mathrm{SSN}=0$, which has also important consequences for the long-term behaviour of TSI. On the other hand, Fröhlich (2009) showed that $B_{R}$, the radial component of $B$, at minima was the only parameter, which could explain the large change of TSI during the last two minima, even though there was no obvious reason for this correlation. Now it is clear why the $\sqrt{\mathrm{SSN}}$ is an excellent proxy for the study of the long-term variability of TSI.

The time series of TSI and $\sqrt{\mathrm{SSN}}$ are low-pass-filtered with a 355-day triangular running mean (indicated as $\langle\cdots\rangle$ ) before they are correlated. The daily time-dependent uncertainty may now be defined as the root-mean-square (rms) deviation of the daily $\left\langle\mathrm{TSI}-S_{0}\right\rangle-f(\langle\sqrt{\mathrm{SSN}}\rangle)$ with $f$ the functional behaviour, which can be determined from a fit to a selection of TSI records versus $\sqrt{\mathrm{SSN}}$. This reference may also be called a "model".

\subsection{TSI data sets}

The following TSI time series are used in this analysis:

- HF org ${ }^{2}$ described in Hoyt et al. (1992). In the Earth Radiation Budget experiment for NIMBUS-7 (first flown on NIMBUS-6), the thermopile detector in channel 10C was replaced by an electrically calibrated cavity radiometer - called Hickey-Frieden (HF, the names of the originators). However, there was no room for a second radiometer, which could have been used as a backup to correct for degradation during the mission. The total exposure time of HF at the end of the over 5000-day-long mission was superior to 150 days. Consequently, HF org shows substantial and still uncorrected long-term changes.

- ERBS org (Earth Radiation Budget Satellite), described in Lee et al. (1987). The radiometer of ERBS is an ACRIM type, but has no backup, as it was planned to be used very rarely. Indeed, the exposure time after the 18.7-year mission was only 2.7 days; so, degradation is not really a problem, but the early increase as observed by the ACRIM-type radiometer during the first few days of exposure still needs to be corrected.

- ACRIM1 org (Active Cavity Radiometer for Irradiance Monitoring [ACRIM] on the NASA Solar Maximum Mission [SMM]) described in Willson (1984). ACRIM1 is originally corrected for degradation, which however overestimates the effect during the spin-mode period with only little exposure time. This period started in December 1980

\footnotetext{
2 "org" is added for the original, as published time series in order to distinguish them from those corrected by Fröhlich (2006), indicated as "corr". If no corrected time series is available, no "org" is added.
}

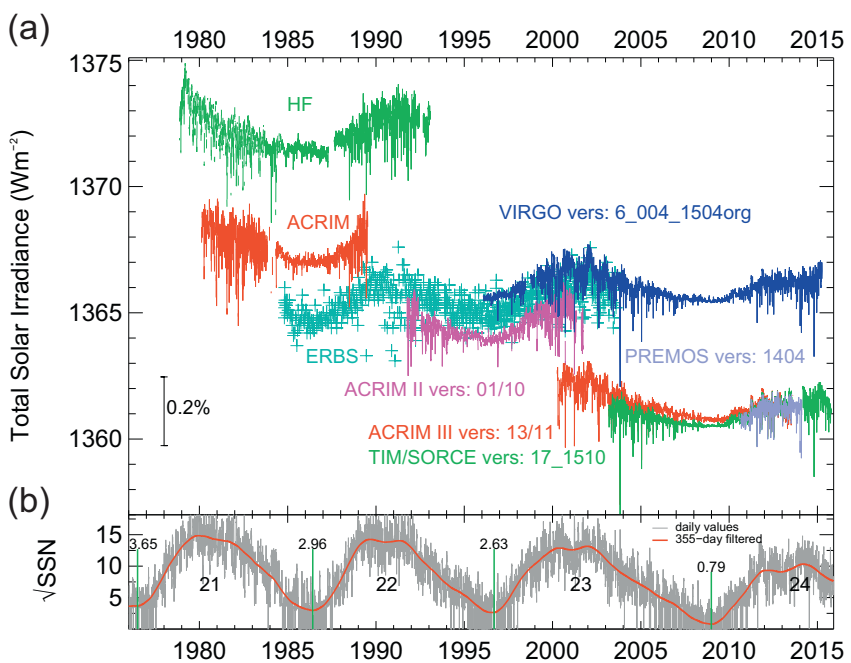

Fig. 1. (a) Compared are daily values of the Sun's total irradiance from radiometers on different space platforms as published by the instrument teams since November 1978. VIRGO is plotted on its original scale and ACRIM3 on the corrected scale of Willson (2014); VIRGO can be reduced to its new scale by multiplying the values by 0.996381 , which brings it to the level of TIM. (b) Daily values of the square root of the sunspot number $(\sqrt{\mathrm{SSN}})$ which will be used in the following analysis. Note the changes of the minima values indicated on top of the vertical lines at their time, especially for the recent one.

after a failure of the momentum wheels used for the 3-axis stabilization of the spacecraft, and lasted until 1984, when the SMM satellite was repaired in space during a shuttle mission.

- ACRIM2 (on the NASA Upper Atmospheric Research Satellite [UARS], data used until 2 Sep 2000, when ACRIM3 took over), described in Willson (1994).

- ACRIM3 (on the NASA ACRIMSat mission, data version 11/13, but only until 2 Feb 2013 as later data seem to be less reliable), described in Willson (2014).

- VIRGO (version 6.4, a combination of the results from the DIARAD and PMO6 V radiometers within the Variability of IRradation and Gravity Oscillations [VIRGO] experiment on the ESA/NASA SOlar and Heliospheric Observatory [SOHO]), described in Fröhlich et al. (1997).

- TIM (Total solar Irradiance Monitor on the NASA SOlar Radiation and Climate Experiment (SORCE), version 17), described in Kopp et al. (2005).

- PREMOS (PREcision MOnitoring Sensor on the French mission PICARD), described in Schmutz et al. (2013).

As mentioned in Section 1, Fröhlich (2006) developed corrections for ACRIM1 including the early increase and the low exposure time during the spin mode. So, ACRIM1 corr could then be used as reference for the missing backup radiometer for HF, which allowed the determination of the corrections for an early increase, the exposure-time-dependent degradation and the non-exposure-dependent sensitivity increase, which was also observed in the behaviour of DIARAD on VIRGO/ SOHO. This increase is related to common features of the construction of these two radiometers, which is quite different from the others. Furthermore, the results of the early-increase correction of ACRIM1 can also be used to correct the early increase of ERBS. These corrected data sets are called HF corr, 


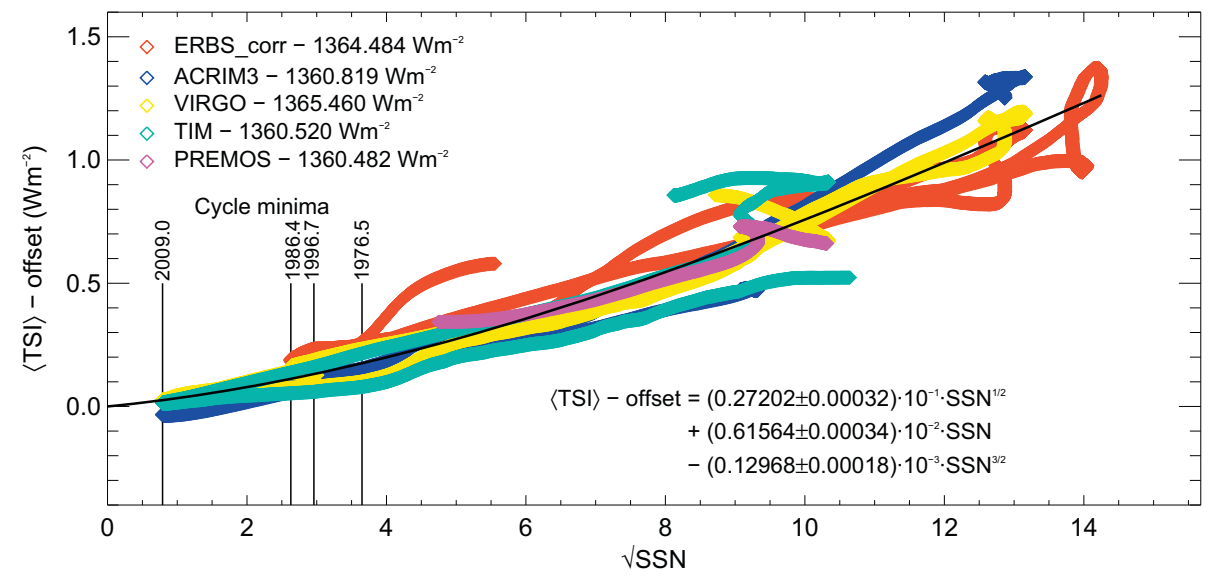

Fig. 2. Scatter plot of $\langle\sqrt{\mathrm{SSN}}\rangle$ versus $\langle$ TSI $\rangle$ represented by ERBS corr, ACRIM3, VIRGO, TIM and PREMOS. The parameters of the polynomial fit used in subsequent analysis are also indicated on the plot. The values of the minima of $\sqrt{\mathrm{SSN}}$ from Figure 1 are also presented.

ACRIM1 corr and ERBS corr, and are also used in the following analysis. The corrections for ACRIM2 determined by Fröhlich (2006) do not change the long-term behaviour substantially, so we use the ACRIM2 org record, but reduce it to the time when ACRIM3 took over (2 Sep 2000) and call it ACRIM2. As the HF and ACRIM1 records start only during the maximum of solar cycle 21 , data from the SATIRE-S model from 23 Aug 1974 to 2 Jul 1987 are added in order to provide a record covering all of solar cycle 21 . The SATIRE-S model (e.g. Ball et al. 2014) is based on the detailed analysis of magnetograms to determine the area of bright features as e.g. faculae, and from the white-light images the area of umbra and penumbra of sunspots. This data set covers the period from the start of the Kitt Peak magnetograms in 1974 to present, ${ }^{3}$ and here we use only the data from 1974 to 1987 to cover cycle 21 .

\subsection{Determination of the reference functional behaviour}

To determine a reference functional behaviour of $\langle$ TSI $\rangle$ versus $\langle\sqrt{\mathrm{SSN}}\rangle$ the time series of ERBS corr, ACRIM3, VIRGO, TIM and PREMOS are used as an ensemble. The daily values of $\left\langle\mathrm{TSI}_{i}\right\rangle-S_{0 i}$ of each series $i$ are adjusted to a third-order polynomial of $\langle\sqrt{\mathrm{SSN}}\rangle$, namely

$$
\begin{aligned}
\left\langle\mathrm{TSI}_{i}\right\rangle-S_{0, i}= & a_{1}\langle\sqrt{\mathrm{SSN}}\rangle+a_{2}\langle\sqrt{\mathrm{SSN}}\rangle^{2} \\
& +a_{3}\langle\sqrt{\mathrm{SSN}}\rangle^{3} .
\end{aligned}
$$

The offset $S_{0, i}$ is estimated for each individual instrument $i$, whereas the polynomial coefficients $a_{1 \ldots 3}$ are determined from the ensemble. Figure 2 shows the result together with the values of the coefficients of the fit and the individual offsets $S_{0 i}$. The relation has a rather high linear coefficient as suggested by Wang \& Sheeley (2003), and the quadratic and cubic terms describe the difference between $\langle\mathrm{TSI}\rangle$ and $\langle\sqrt{\mathrm{SSN}}\rangle$. This is mainly due to the stronger response of TSI to more and larger spots and faculae with increasing activity. This effect depends on the net effect of the sunspot darkening and facular brightening, and may change from cycle to cycle: the mean of the net effect over about 3 years around the maximum of cycles 21, 22, 23 and 24 amounts to $-0.27,-0.12,-0.04$ and $+0.17 \mathrm{Wm}^{-2}$ (calculated with the

\footnotetext{
3 Available from http://www.mps.mpg.de/projects/sun-climate/ data.html
}

proxy model of Fröhlich 2011), which changes the cycle amplitudes between $-20 \%$ and $+15 \%$. Thus, the higher-order terms of the polynomial should increase from cycle 21 to 23 , which is visible in the results of ACRIM1 corr, ERBS corr and ACRIM2. This in turn suggests that the reference should deviate subsequently less from the linear behaviour towards higher values. For cycle 24 the deviations at around the maximum are even more important, as shown by VIRGO, TIM and PREMOS (Figs. A.7-A.9). All this shows that the $\langle\sqrt{\mathrm{SSN}}\rangle$ is explaining well the intra-cycle changes of $\langle\mathrm{TSI}\rangle$ as represented by the values during minima.

\subsection{Results for all TSI records}

The results of the comparison of $\sqrt{\mathrm{SSN}}$ with all the different TSI data sets are presented in Figures 3 and 4, A.1-A.10, and are summarized in Table 1. Figures $3 \mathrm{a}$ and $4 \mathrm{a}$ show the colour-coded temporal evolution of the daily $\langle$ TSI $\rangle-S_{0}$ relative to $\langle\sqrt{\mathrm{SSN}}\rangle$ together with the fitted "reference" (full black line) from Figure 2. Also shown are the values at the minima as indicated in Figure 1. Figure $1 \mathrm{~b}$ shows the colour code used in Figure 1a for the temporal evolution together with the corresponding deviations between TSI and the reference. These are calculated as standard deviations of 81-day intervals and provide (i) the signed deviations, which are used to characterize the "trend", which may not be linear and is hence characterized by a quadratic fit (full red line on the plots), which is also characterized by a linear trend (dashed red line) and a curvature (presented in Table 1); (ii) the rms of the signed (full blue line) and the average (dashed blue line), which provides an estimate for the instrumental and operationally influenced uncertainty; (iii) the standard deviation and a quadratic fit to it (full green line), which finally defines the time-dependent uncertainty, including trends, manifested by increasing values towards both ends.

The change of the deviations between the original and the corrected HF records (Figs. 3 and 4) is impressive. The large curvature in the original record is due to the two different effects of the degradation and sensitivity increase. The corrected one is also curved, which is similar to ACRIM1 corr used as reference for the HF corrections. This effect is possibly due to uncorrected effects of ACRIM1 during the spin mode of SMM (Dec 1980-Feb 1984). The mean rms deviation, a measure of the noise of the measurements of HF corr, is with 
(a)

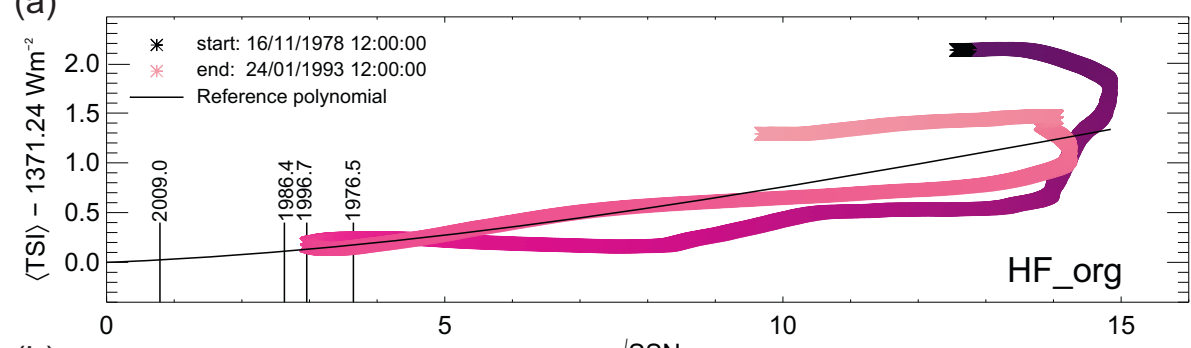

(b)

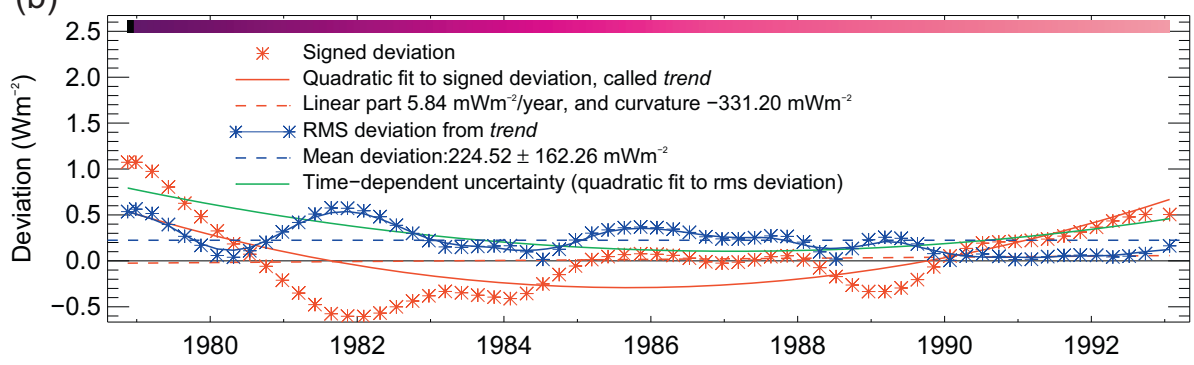

Fig. 3. Results for HF org. (a) The scatter plot of $\langle\sqrt{\mathrm{SSN}}\rangle$ versus $\langle\mathrm{TSI}\rangle$ and (b) the results of the deviation analysis. Also shown is the time dependence of the colour used in (a) (for details see Sect. 2.3). The large deviation confirms the missing corrections for the degradation and the non-exposure-dependent sensitivity increase, which show as a decrease of the "trend" until about 1986 and an increase after.

(a)

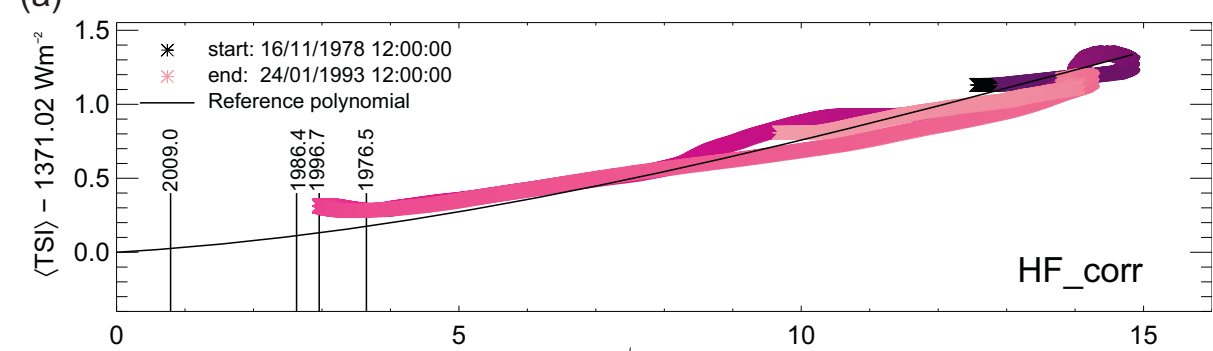

(b)

$\sqrt{S S N}$

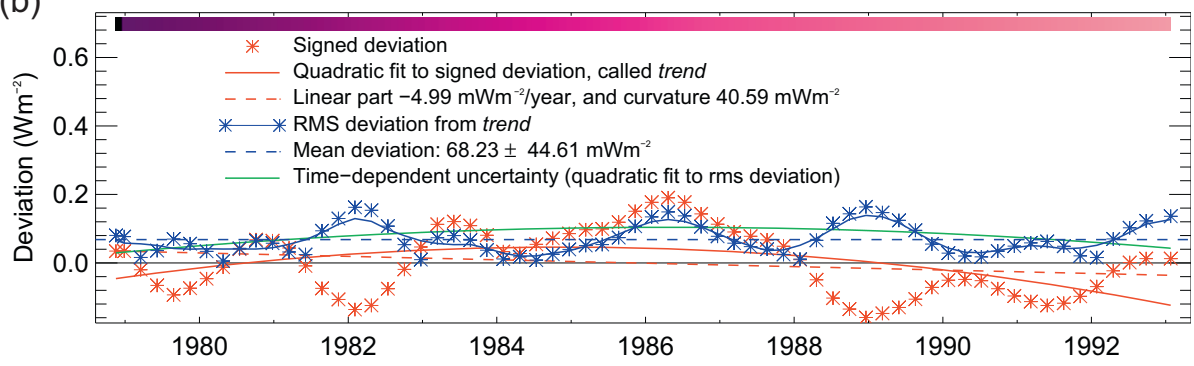

Fig. 4. Results for HF corr (for explanation of the plots see Fig. 3 and Sect. 2.3). The corrections of HF have been determined by Fröhlich (2006). The reduction of the large deviation at the beginning and end of the mission is impressive. The upward curved trend is similar to ACRIM1 corr, which was used as reference for the HF corrections, and hence not a feature of this cycle.

$68 \mathrm{mWm}^{-2}$ much higher than the average of about $45 \mathrm{mWm}^{-2}$. ERBS corr has an even higher rms deviation with $86 \mathrm{mWm}^{-2}$. For both experiments this is due to the very short observations during 5 min every 90-minute orbit for HF and every 14 days for ERBS.

A modulation of the deviation with a period of about two years and with increasing amplitude during higher levels of solar activity is apparent on most of the records. This effect is likely due to the modulation of the annual distance variation by differences of the activity manifestations in the two solar hemispheres. Due to the inclination of the solar rotation axis, the latitude of the centre of the observed disc is oscillating between of $\pm 7.25^{\circ}$ with a period of half a year. Because the sunspots are located in a latitude band of about $\pm 40^{\circ}$ the sum of their numbers will not change, even if their amount in each hemisphere is quite different. For TSI all features on the solar disc contribute, and hence, the asymmetry modulates the annual distance variation and produces side bands at \pm 0.5 years. With the correction of the distance (to refer TSI to $1 \mathrm{AU}$ ) the 1-year modulation is removed and only the side bands remain, which produce a 2-year oscillation. It is interesting that SATIRE-S also shows this 2-year modulation, which suggests that magnetograms are also sensitive to the asymmetry of the two hemispheres. Other solar effects may disturb this oscillation so that it may be less clear or even hidden. Anyway, the mean rms deviation includes solar effects, which are not instrument related and so it is an upper limit of the uncertainty. 


\section{Fröhlich: Time-dependent uncertainty of the total solar irradiance}

Table 1. Summary of the properties of the time series used in this analysis. The characteristics of the comparison with $\langle\sqrt{\mathrm{SSN}}\rangle$ are summarized as: "Mean rms", which is the average of the rms deviation from the trend, presented as "Slope", which is the result of the linear fit to the trend (given as per year) and as "Curvature", which is the maximal distance of the curved trend from the straight line through the end points, divided by the length of the record in years.

\begin{tabular}{|c|c|c|c|c|c|c|c|}
\hline & Start date & End date & $\begin{array}{c}\text { Duration } \\
\text { days }\end{array}$ & $\begin{array}{c}\text { Offset } \\
\left(\mathrm{Wm}^{-2}\right)\end{array}$ & $\begin{array}{l}\text { Mean rms } \\
\left(\mathrm{mWm}^{-2}\right)\end{array}$ & $\begin{array}{c}\text { Slope } \\
\left(\mathrm{mWm}^{-2} / \mathrm{a}\right)\end{array}$ & $\begin{array}{c}\text { Curvature } \\
\left(\mathrm{mWm}^{-2} / \mathrm{a}\right)\end{array}$ \\
\hline HF org & 16 Nov 1978 & 24 Jan 1993 & 5183 & 1371.238 & 224.5 & 5.84 & -23.340 \\
\hline HF corr & 16 Nov 1978 & 24 Jan 1993 & 5183 & 1371.022 & 68.23 & -4.99 & 2.860 \\
\hline ACRIM1 org & 16 Feb 1980 & 14 Jul 1989 & 3436 & 1366.794 & 97.34 & -15.45 & 2.150 \\
\hline $\begin{array}{l}\text { ACRIM1 } \\
\text { corr }\end{array}$ & 16 Feb 1980 & 14 Jul 1989 & 3436 & 1366.779 & 45.99 & 3.10 & 7.629 \\
\hline ERBS org & 24 Oct 1984 & 5 Aug 2003 & 6859 & 1364.726 & 88.07 & 19.96 & -1.150 \\
\hline ERBS corr & 24 Oct 1984 & 5 Aug 2003 & 6859 & 1364.484 & 85.52 & -5.64 & -1.476 \\
\hline ACRIM2 & 5 Oct 1991 & 2 Sep 2000 & 3255 & 1363.838 & 30.58 & 29.39 & 0.796 \\
\hline VIRGO & 28 Jan 1996 & 8 Apr 2015 & 7010 & 1365.460 & 41.36 & -2.54 & -0.466 \\
\hline ACRIM3 & 5 Apr 2000 & 3 Feb 2013 & 4687 & 1360.819 & 47.05 & -31.87 & -1.301 \\
\hline TIM & 4 Mar 2003 & 13 Oct 2015 & 4606 & 1360.520 & 51.86 & 36.67 & -2.075 \\
\hline PREMOS & 27 Jul 2010 & 11 Feb 2014 & 1295 & 1360.482 & 49.67 & 9.18 & 3.748 \\
\hline SATIRE-S & 23 Aug 1974 & 2 Jul 1987 & 4696 & 1360.834 & 83.38 & -3.45 & -4.627 \\
\hline
\end{tabular}

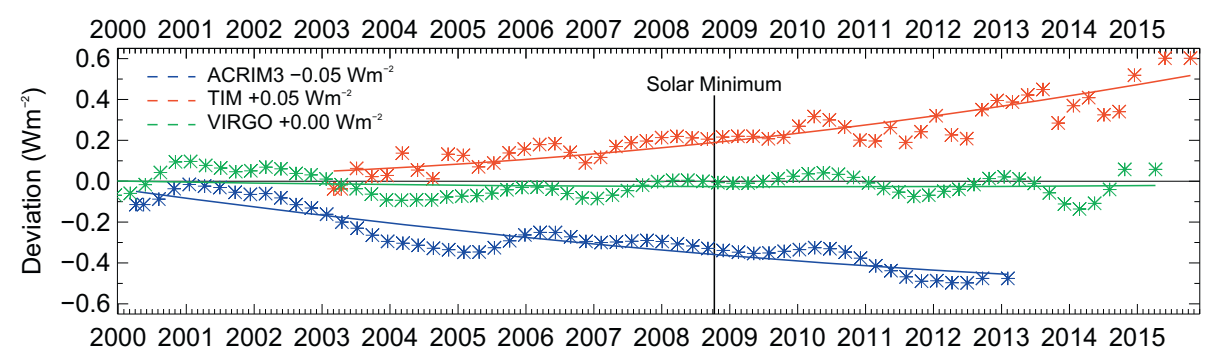

Fig. 5. Shown are the trends of ACRIM3 and TIM, compared to VIRGO from the results of the deviation analysis of Figures A.6, A.8 and A.7. The trends of ACRIM3 and TIM are quite large, indicating a problem with the degradation correction. All three show similar temporal variation of the rms deviation from the trend. Hence, the deviations common in all records are due to solar effects and not instrumental.

A comparison of the trends of VIRGO, ACRIM3 and TIM with their rms deviations is shown in Figure 5. These trends are determined as deviations from the reference polynomial, which explains the trend of VIRGO as being with $-2.5 \mathrm{mWm}^{-2} / \mathrm{a}$ close to the average of the slopes of TIM and ACRIM3 of $4.8 \mathrm{mWm}^{-2} / \mathrm{a}$. The curvature is more relevant for the diagnostic of the degradation corrections, which is for VIRGO a factor of 3-4 smaller than the others, indicating again the value of its more sophisticated degradation-correction algorithm. Older versions of ACRIM3 showed a strong annual oscillation, which could be removed by a revision of the temperature correction, the result of which leads to a very similar rms deviation as for VIRGO, but with a higher amplitude. VIRGO has only an annual temperature variation due to the orbit of $\mathrm{SOHO}$ around the Lagrange point, whereas for ACRIM3 the annual temperature effect is on top of the strong temperature modulation due to the orbit around Earth, and hence, this correction introduces also noise. The stronger curvatures of the TIM and ACRIM3 trends indicate problems with their degradation corrections. Although the corrections for TIM are much smaller than those for ACRIM3 the deviation for TIM is much larger, which provides interesting information about the algorithm used to determine the corrections.

The values of the minima shown in Table 2 provide interesting insights into the intra-cycle variations. For those time series, which have only one minimum, the value is the result of the determination of $S_{0}$ during the fitting. More interesting are the results for the two records that include two minima,
Table 2. TSI values during solar minima from the comparison of $\sqrt{\mathrm{SSN}}$ and TSI. The uncertainty presented is the standard deviation of the 3-4 determinations and is only a measure of the differences between the records. ERBS corr and VIRGO records cover two minima, which allow the comparison of the overall trends from Table 1 with the change between the observed minima.

\begin{tabular}{llll}
\hline \hline Minimum & Data & \multicolumn{2}{c}{ Values at minimum } \\
\cline { 3 - 4 } & & TSI $\left(\mathrm{Wm}^{-2}\right)$ & $\sqrt{\mathrm{SSN}}$ \\
\hline 1976.50 & SATIRE-S & 0.239 & 3.649 \\
1986.42 & HFcorr & 0.314 & \\
& ERBS corr & 0.148 & \\
& ACRIM1 corr & 0.253 & \\
& SATIRE-S & 0.250 & \\
1996.69 & Average & $0.229 \pm 0.060$ & 2.959 \\
& ERBS corr & 0.191 & \\
& ACRIM2 & 0.117 & \\
& VIRGO & 0.164 & \\
& Average & $0.157 \pm 0.031$ & 2.629 \\
& ACRIM3 & -0.031 & \\
& VIRGO & 0.028 & \\
& TIM & 0.008 & 0.789 \\
\hline & Average & $0.002 \pm 0.024$ & \\
& & & \\
\end{tabular}

ERBS corr with the minima at 1986 and 1998 and VIRGO with those at 1998 and 2008. From the difference between the minima of 0.04 and $0.14 \mathrm{Wm}^{-2}$ for ERBS and VIRGO, respectively, a trend of $3.1 \mathrm{ppm} / \mathrm{a}$ and $-7.4 \mathrm{ppm} / \mathrm{a}$ is 
(a)

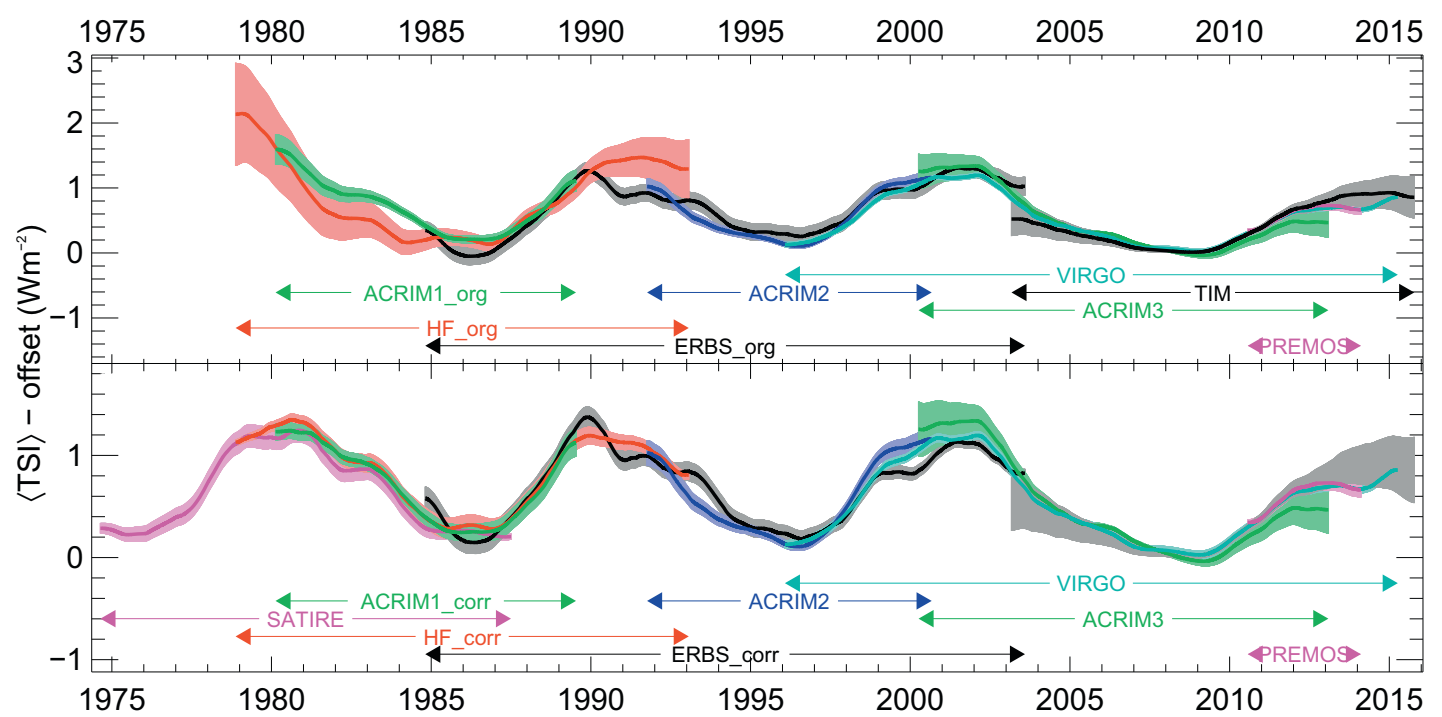

Fig. 6. Shown are all TSI time series (full line) with their uncertainty (green curve of the individual TSI plots, shown as shaded area), in (a) with the "org" and (b) the "corr" data sets. Note the difference in scale by a factor of 1.6 needed to include HF org. The determined offsets are used to shift the individual time series to a common level. Moreover, the SATIRE-S model provides a useful extension back to 1975 and supports the corrections of HF and ACRIM1 during solar cycle 21.

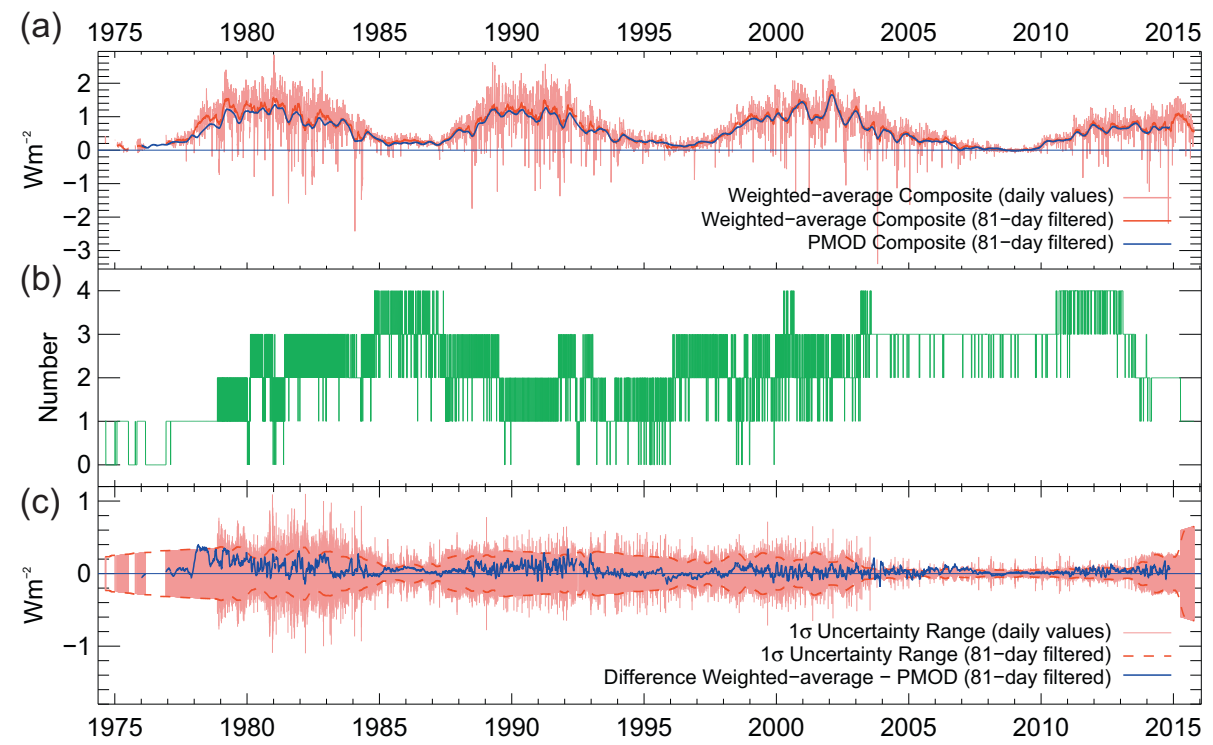

Fig. 7. (a) Daily values of a weighted-average and the 81-day filtered "new" composite (red) and for comparison the 81-day filtered PMOD composite (blue). (b) Number of records used for each daily value and (c) the rms deviation of the weighted average and the difference between the two composites. Note the jump during 1978, which is due to an incorrect extension of the PMOD composite with the results of a proxy model.

determined, which can be compared to the trend calculated over the full time series of $-4.2 \mathrm{ppm} / \mathrm{a}$ and $-1.9 \mathrm{ppm} / \mathrm{a}$ (Table 1). The difference of $-7.2 \mathrm{ppm} / \mathrm{a}$ and $5.5 \mathrm{ppm} / \mathrm{a}$ may be used as a measure of the long-term uncertainty. The large difference for ERBS may be still due to some missing degradation correction at the beginning of its mission and so an uncertainty of about $\pm 6 \mathrm{ppm} / \mathrm{a}$ or $\pm 65 \mathrm{ppm}\left(0.09 \mathrm{Wm}^{-2}\right)$ per cycle may be estimated and as a rms sum of $\pm 125 \mathrm{ppm}$ $\left(0.17 \mathrm{Wm}^{-2}\right)$ for the full period from 1976 to present.

The individual time-dependent uncertainties from the comparison with $\sqrt{\mathrm{SSN}}$ are presented in Figure 6a for all the original records and in Figure $6 \mathrm{~b}$ together with the corrected ones. This shows that the present analysis provides an investigatorindependent estimation of the uncertainty, which may indeed be used for weighting the records into a composite - at least for longer-term trends (over more than 1 year), which is anyway the most important aspect of a TSI composite. Figure $6 \mathrm{~b}$ supports the idea that a reliable composite for the three and half solar cycles can indeed be constructed by using the corrected series instead of the original ones.

\section{Construction of a "new" composite}

The results of Figure $6 \mathrm{~b}$ can be used to construct a TSI composite by a weighted average of the daily values of the available records with $1 / \sigma^{2}$ as weights, with $\sigma$ the values of the quadratic fit to the overall standard deviation (green curve) of the 
corresponding record. The result of this simple approach is shown in Figure 7, and indeed it compares well with the PMOD composite. This is, however, not astounding as the PMOD composite is built from the same records, but with one record at a time and adjusting them on overlapping periods. Looking at Figure $7 \mathrm{c}$ it becomes clear that such an approach needs some refinement, which has to make sure that the overall long-term variability is maintained. When a new record starts or ends, then the value and the standard deviation change immediately and a new adjustment would be needed. There are some other problems as e.g. the jump in 1978 (Fig. 7c), which may indicate that the proxy model used to extend the PMOD composite may be wrongly adjusted and scaled due to the problem of the ACRIM1 corrections during the spin mode of SMM. Although the general agreement is surprisingly good it is certainly not a competitive alternative, but still interesting as a diagnostic tool. A far better method is described by Dudok de Wit (2011) with which all records can be extrapolated to the full length before they are joined. The time-dependent long-term uncertainty presented here will then improve the reliability of such a composite even further.

\section{Conclusion}

The comparison of TSI and $\sqrt{\mathrm{SSN}}$, both low-pass-filtered with a 13-Carrington-rotation-long triangular running mean, proves to be a valuable method to determine a time-dependent uncertainty from the rms deviation of the residual error between TSI and the reference functional behaviour determined by fitting a third-order polynomial to a selection of TSI records. The results are independent of the investigator's judgement and allow a direct comparison of the representativeness of each TSI record. The detailed analysis of the deviations from the reference allows to characterize the behaviour and performance of each record. Trends mean either the existence of a physical trend in TSI, which cannot be reproduced by the $\mathrm{SSN}$, or possible flaws in the algorithms used for the correction of degradation and other long-term changes. Examples are the trends and curvatures of TIM and ACRIM3 in comparison with the very small values of VIRGO, which demonstrates the success of more sophisticated corrections. Furthermore, including the time series from the SATIRE-S model from 1974 to 1987 does not only enhance the value of a new composite for studies of TSI variability, but also improves the reliability of the maximum of solar cycle 21 . In conclusion, the $\sqrt{\mathrm{SSN}}$ is a very interesting proxy for TSI as it describes the photospheric magnetic field at minima very well (as shown by e.g. Wang \& Sheeley 2003), and provides a hint of what lowest values TSI at minima may be reached on century timescales, namely the "floor" of Svalgaard \& Cliver (2007).

All daily time series with their uncertainties (green curves in the lower panel of the TSI figures) are available as ascii (.dat) or IDL-save files (.idl) - accordingly named - from the PMOD/WRC ftp server in the directory ftp:// ftp.pmodwrc.ch/pub/Claus/TSI-uncertainty/.

Acknowledgements. The idea for this investigation came up during the last meeting of the ISSI international team "An Assessment of the Accuracies and Uncertainties in the Total Solar Irradiance Climate Data Record" in May 2014, and I would like to thank the team members for their support, especially Greg Kopp from LASP, Boulder, CO, USA, the leader of the team. Thanks are extended to Thierry Dudok de Wit, LPC2E/CNRS, Orléans, France, for helpful discussions about the statistics and the construction of
TSI composites. Finally I would like to thank travel support from the Swiss National Science Foundation through a PMOD/WRC project. Furthermore, the comments of three referees, which improved the paper and its understandability substantially, are gratefully acknowledged. The editor thanks Laure Lefèvre and an anonymous referee for their assistance in evaluating this paper.

\section{References}

Abbot, C.G. Measurements of solar radiation. Astrophys. J., 37, 130, 1913, DOI: 10.1086/141982.

Ångström, A. Solar constant, sun-spots and solar activity. Astrophys. J., 55, 24, 1922, DOI: 10.1086/142654.

Anscombe, F. The transformation of Poisson, binomial and negative-binomial data. Biometrika, 35, 246-254, 1948.

Ball, W.T., Y.C. Unruh, N.A. Krivova, S. Solanki, T. Wenzler, D.J. Mortlock, and A.H. Jaffe. Reconstruction of total solar irradiance 1974-2009. Astron. Astrophys., 541, A27, 2012, DOI: 10.1051/0004-6361/201118702.

Ball, W.T., N.A. Krivova, Y.C. Unruh, J.D. Haigh, and S.K. Solanki. A new SATIRE-S spectral solar irradiance reconstruction for solar cycles 21-23 and its implications for stratospheric ozone. J. Atmos. Sci., 71, 4086-4101, 2014, DOI: 10.1175/JAS-D-13-0241.1.

Clette, F., L. Svalgaard, J.M. Vaquero, and E.W. Cliver. Revisiting the sunspot number. A 400-year perspective on the solar cycle. Space Sci. Rev., 186, 35-103, 2014,

DOI: $10.1007 / \mathrm{s} 11214-014-0074-2$.

Dewitte, S., D. Crommelynck, S. Mekaoui, and A. Joukoff. Measurement and uncertainty of the long-term total solar irradiance trend. Sol. Phys., 224, 209-216, 2004, DOI: $10.1007 / \mathrm{s} 11207-005-5698-7$.

Dudok de Wit, T. A method for filling gaps in solar irradiance and solar proxy data. Astron. Astrophys., 533, A29, 2011, DOI: $10.1051 / 0004-6361 / 201117024$.

Fröhlich, C. Solar irradiance variability since 1978: revision of the PMOD composite during solar cycle 21. Space Sci. Rev., 125, 53-65, 2006, DOI: 10.1007/s11214-006-9046-5.

Fröhlich, C. Evidence of a long-term trend in total solar irradiance. Astron. Astrophys., 501, L27-L30, 2009, DOI: 10.1051/0004-6361/200912318.

Fröhlich, C. A four-component proxy model for total solar irradiance calibrated during solar cycles 21-23. Contrib. Astron. Obs. Skalnate Pleso, 41, 113-132, 2011.

Fröhlich, C. Total solar irradiance observations. Surv. Geophys., 33, 453-473, 2012, DOI: 10.1007/s10712-011-9168-5.

Fröhlich, C., D. Crommelynck, C. Wehrli, M. Anklin, S. Dewitte, et al. In-flight performances of VIRGO solar irradiance instruments on SOHO. Sol. Phys., 175, 267-286, 1997, DOI: $10.1023 / \mathrm{A}: 1004929108864$

Hoyt, D.V., H.L. Kyle, J.R. Hickey, and R.H. Maschhoff. The NIMBUS-7 solar total irradiance: a new algorithm for its derivation. J. Geophys. Res., 97, 51-63, 1992.

Kopp, G., G. Lawrence, and G. Rottman. The Total Irradiance Monitor (TIM): science results. Sol. Phys., 230, 129-139, 2005, DOI: $10.1007 / \mathrm{s} 11207-005-7433-9$.

Lee III, B.R. Barkstrom, R.B., and R.D. Cess. Characteristics of the earth radiation budget experiment solar monitors. Appl. Opt., 26, 3090-3096, 1987.

Schmutz, W., A. Fehlmann, W. Finsterle, G. Kopp, and G. Thuillier. Total solar irradiance measurements with PREMOS/PICARD. In: R.F. Cahalan, and J. Fischer, Editors. IRS2012: Proceedings of the International Radiation Symposium (IRC/IAMAS), 1531, American Institute of Physics, AIP Conf. Proc, Melville, NY, USA, 624-627, 2013.

Svalgaard, L., and E.W. Cliver. A floor in the solar wind magnetic field. Astrophys. Lett. Comm., 661, L203-L206, 2007, DOI: $10.1086 / 518786$.

Wang, Y.-M., and N.R. Sheeley. Modeling the Sun's large-scale magnetic field during the maunder minimum. Astrophys. J., 591, 1248-1256, 2003. 
Willson, R.C. Measurements of solar total irradiance and its variability. Space Sci. Rev., 38, 203-242, 1984,

DOI: $10.1007 / \mathrm{BF} 00176830$.

Willson, R.C.. Irradiance observations from SMM, UARS and ATLAS experiments. In: J. Pap, C. Fröhlich, H.S. Hudson, and S. Solanki, Editors. IAU Colloquium No. 143: The Sun as a Variable
Star: Solar and Stellar Irradiance Variations, Cambridge University Press, Cambridge, UK, 54-62, 1994.

Willson, R.C. ACRIM3 and the Total Solar Irradiance database. Astrophys. Space Sci., 352, 341-352, 2014,

DOI: $10.1007 / \mathrm{s} 10509-014-1961-4$.

Cite this article as: Fröhlich C. Determination of time-dependent uncertainty of the total solar irradiance records from 1978 to present. $J$. Space Weather Space Clim., 6, A18, 2016, DOI: 10.1051/swsc/2016012.

\section{Appendix A. Plots of $\langle$ TSI $\rangle$ versus $\langle\sqrt{\text { SSN }}\rangle$ for ACRIM1, ERBS, ACRIM2, ACRIM3, VIRGO, TIM, PREMOS and SATIRE-S}

(a)

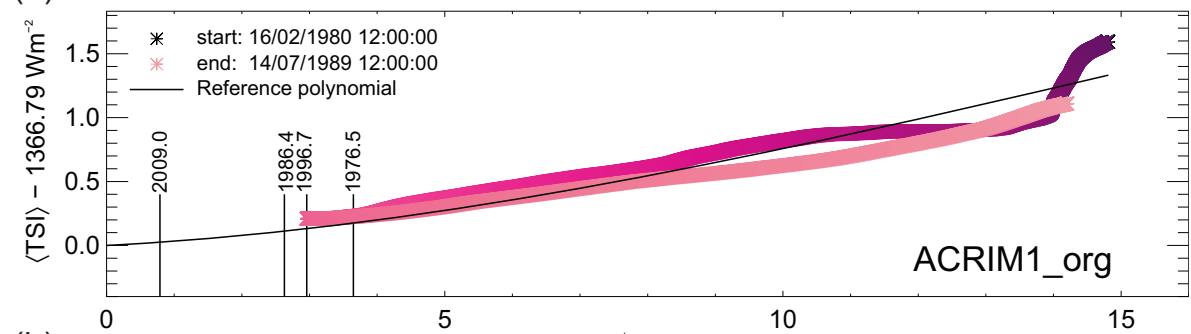

(b)

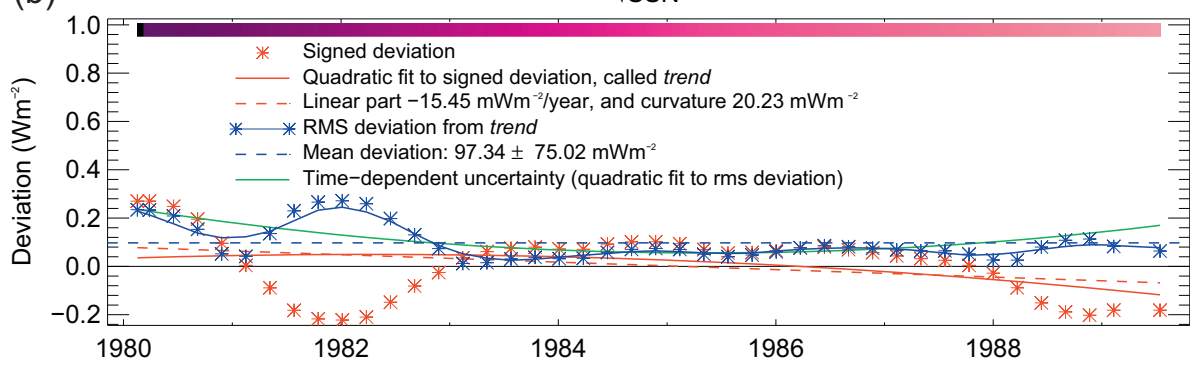

Fig. A.1. Results for ACRIM1 org. (a) The scatter plot of $\langle\sqrt{\mathrm{SSN}}\rangle$ versus $\langle$ TSI $\rangle$ and (b) the results of the deviation analysis. Also shown is the time dependence of the colour used in (a) (for details see Sect. 2.3). The main deviation is during 1980, which means that the degradation correction is inadequate.

(a)

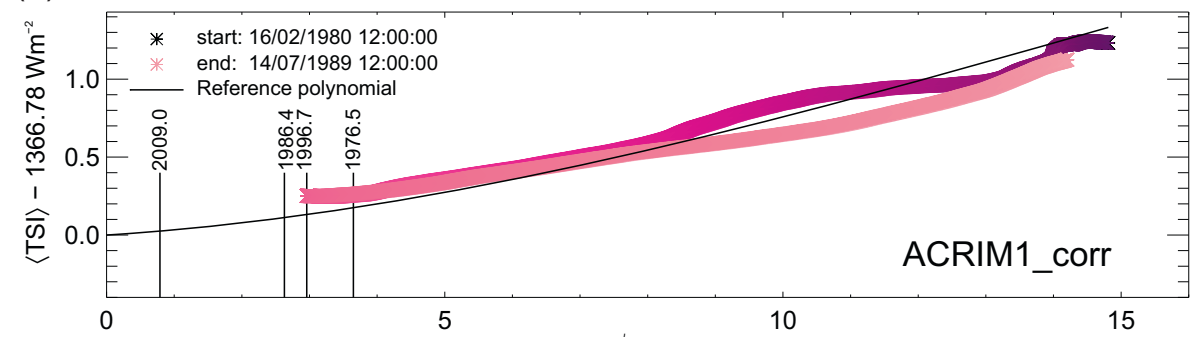

(b)

$\sqrt{S S N}$

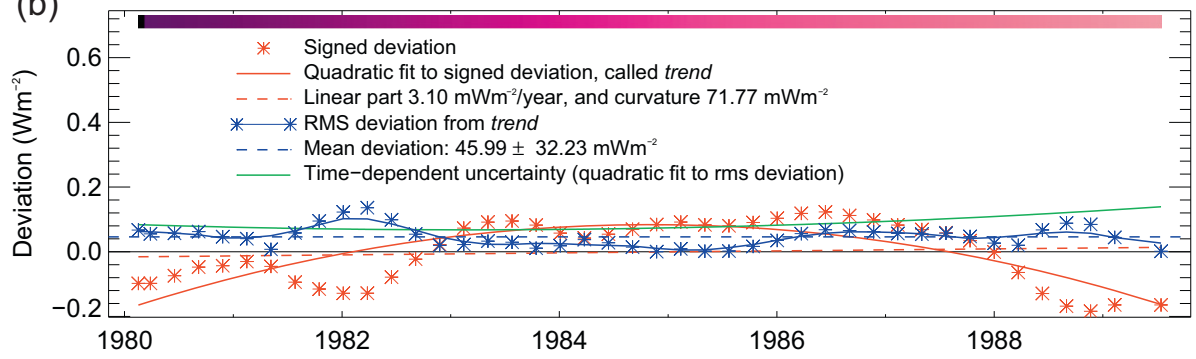

Fig. A.2. Results for ACRIM1 corr (for explanation of the plots see Fig. A.1 and Sect. 2.3). During the spin mode of SMM (Dec 1980-Feb 1984) there seem to be still some problems of degradation, probably due to an inaccurate knowledge of the exposure time. 

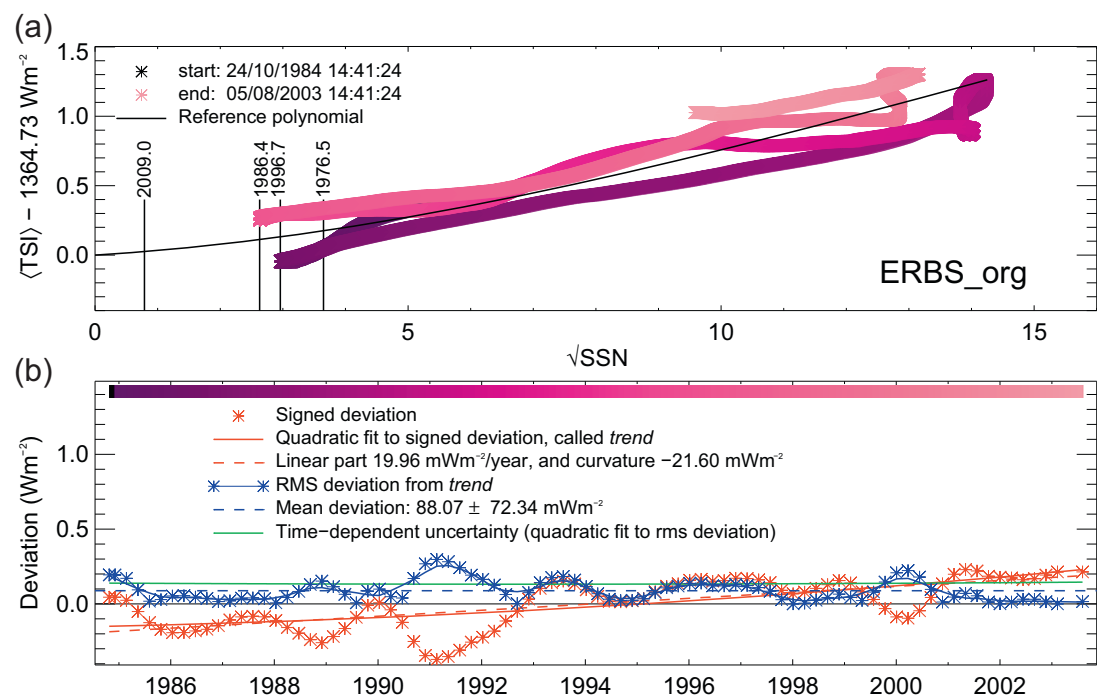

Fig. A.3. Results for ERBS org (for explanation of the plots see Fig. A.1 and Sect. 2.3). This record shows an upward trend by more than $0.5 \mathrm{Wm}^{-2}$ over the whole mission, which is due to the uncorrected early increase.

(a)

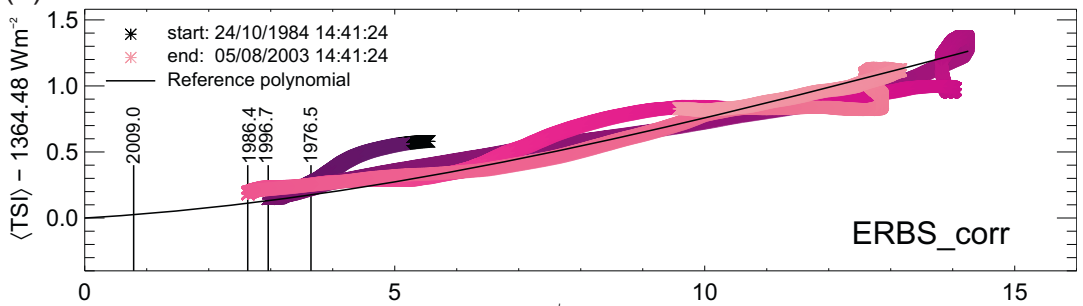

(b)

$\sqrt{S S N}$

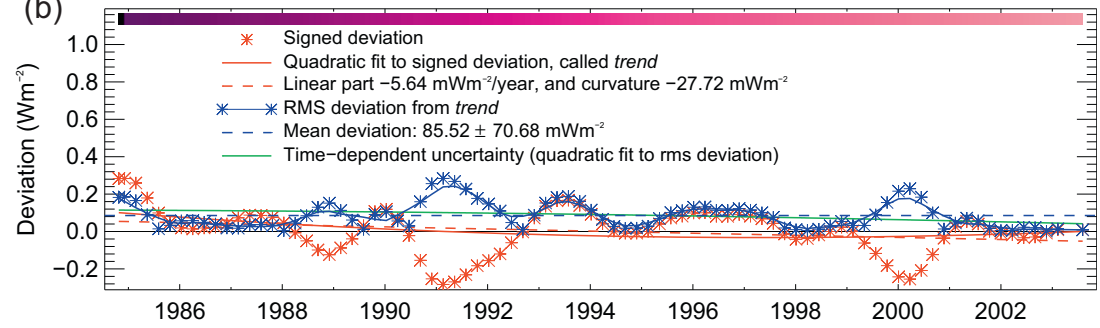

Fig. A.4. Results for ERBS corr (for explanation of the plots see Fig. A.1 and Sect. 2.3). The corrections of ERBS are based on the earlyincrease results of ACRIM1 and seem to correct the trend. At the beginning of the mission the radiometer shows some still uncorrected degradation, possibly due to outgassing within the spacecraft. This effect may also explain the low TSI value at the minimum 1986.
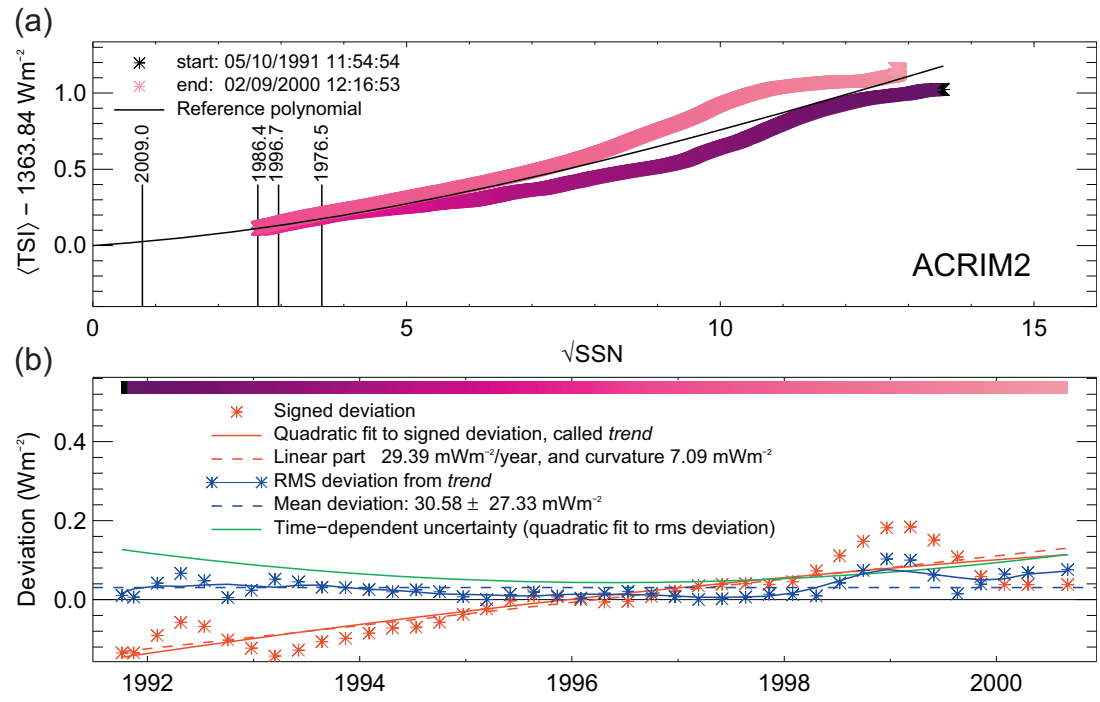

Fig. A.5. Results for ACRIM2 (for explanation of the plots see Fig. A.1 and Sect. 2.3). This record shows still some uncorrected degradation and an influence of the activity level during maxima. 


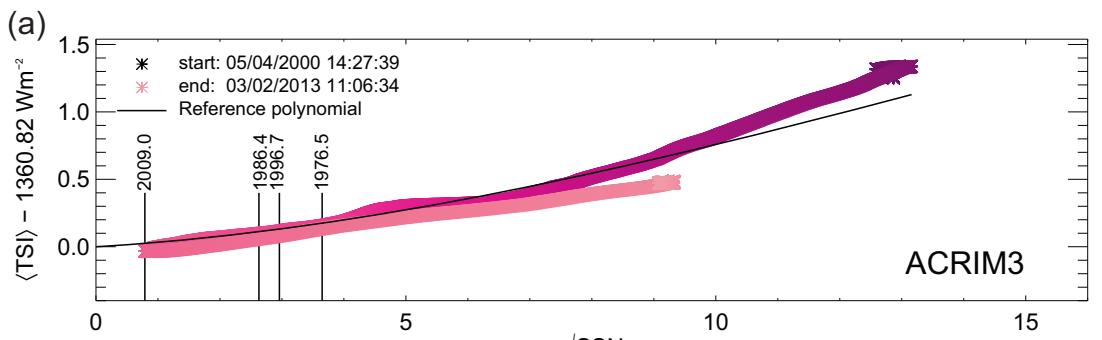

(b)

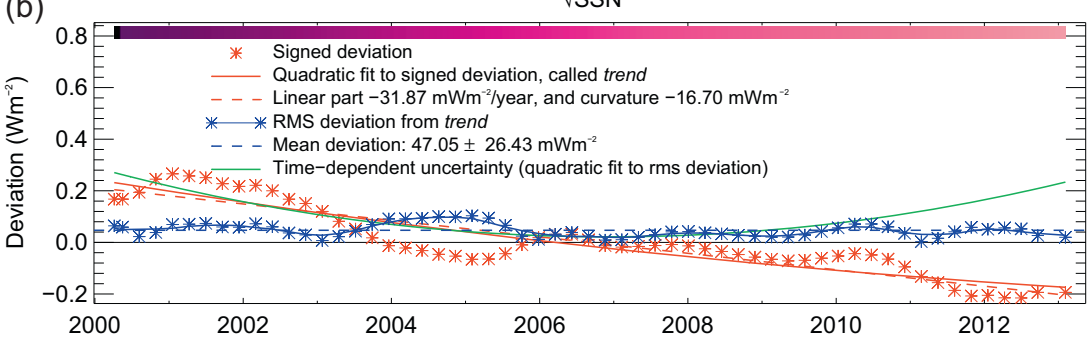

Fig. A.6. Results for ACRIM3 (for explanation of the plots see Fig. A.1 and Sect. 2.3). The overall downward trend is quite important, which implies that the applied corrections for degradation are incomplete (see also Fig. 5).
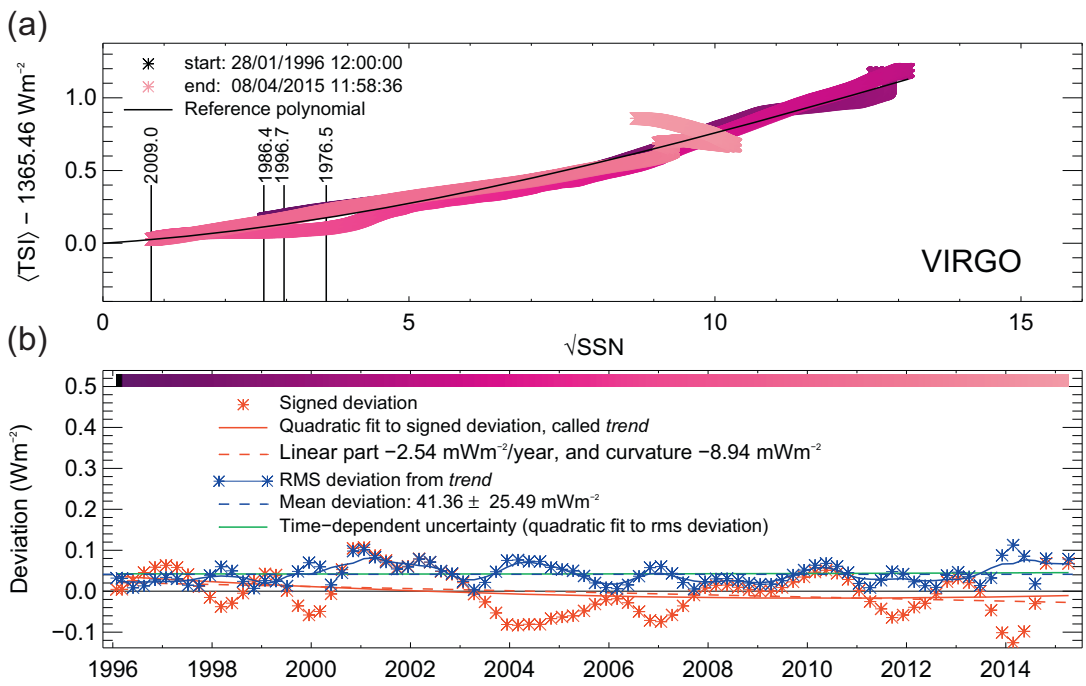

Fig. A.7. Results for VIRGO (for explanation of the plots see Fig. A.1 and Sect. 2.3). Overall, VIRGO has the smallest trend over 20 years, which is remarkable. The deviations during the maximum of cycle 24 are important and present in TIM and PREMOS. This means that they are of solar origin.
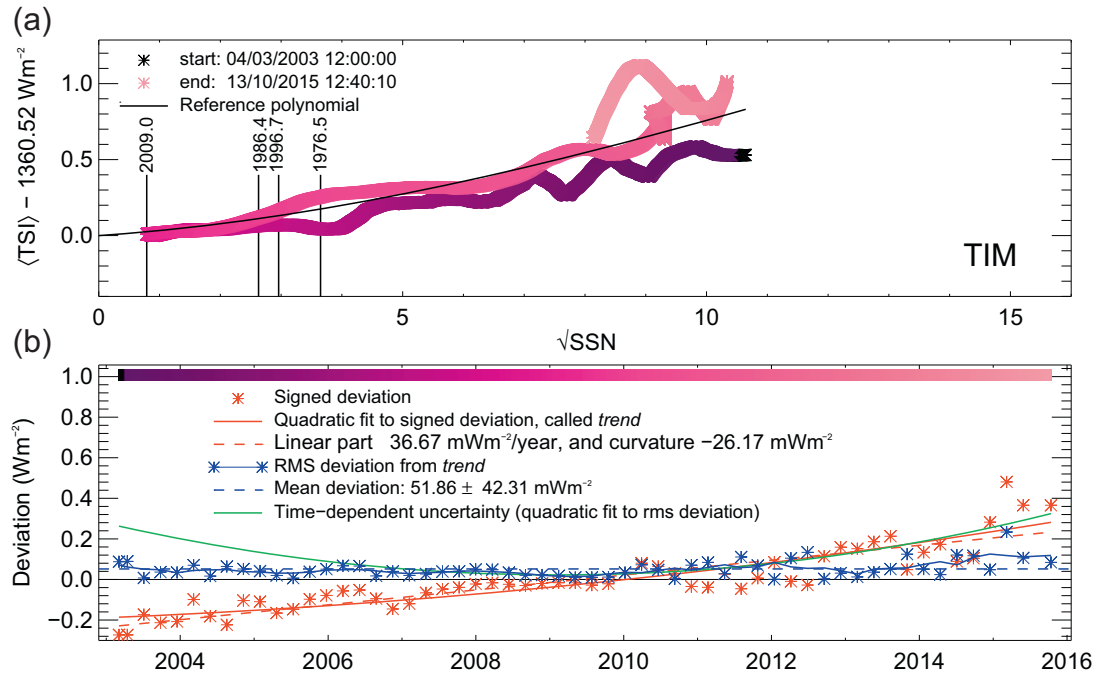

Fig. A.8. Results for TIM (for explanation of the plots see Fig. A.1 and Sect. 2.3). The overall trend is quite important, meaning that there are still some problems with the degradation correction. 
C. Fröhlich: Time-dependent uncertainty of the total solar irradiance
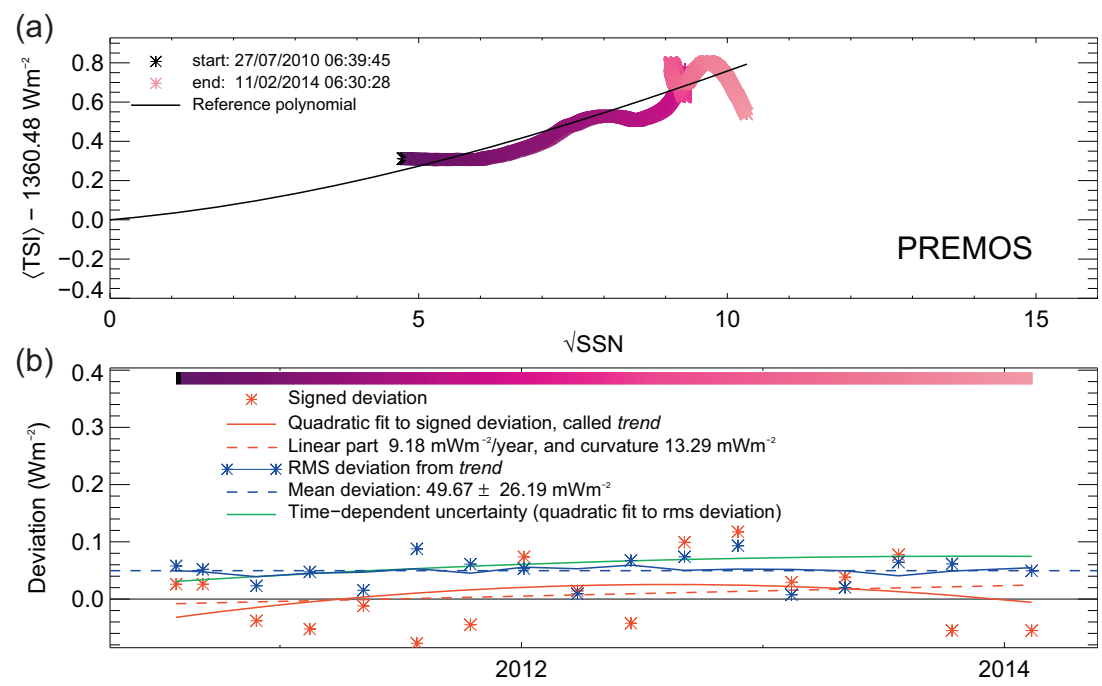

Fig. A.9. Results for PREMOS (for explanation of the plots see Fig. A.1 and Sect. 2.3). This is the shortest record and thus the results are not very conclusive.
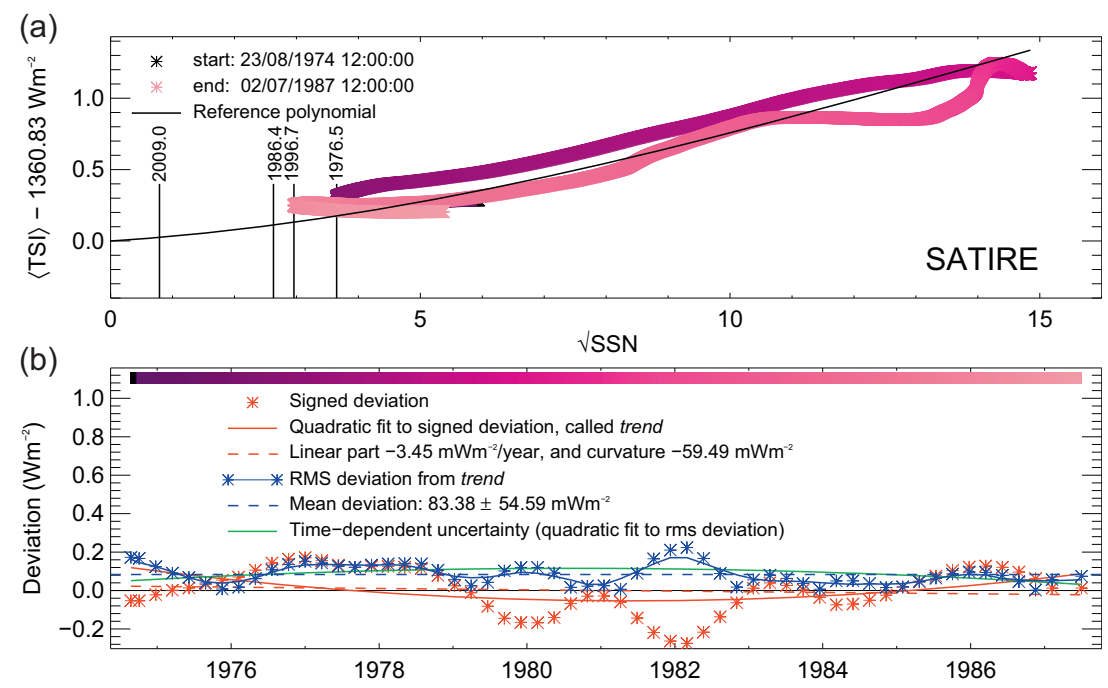

Fig. A.10. Results for SATIRE-S, from Ball et al. 2012 (for explanation of the plots see Fig. A.1 and Sect. 2.3). The large amplitude of the biannual modulation in 1982 is also present in ACRIM1 and hence of solar origin. 